\title{
A Model of Lexical Attraction and Repulsion*
}

\author{
Doug Beeferman Adam Berger John Lafferty \\ School of Computer Science \\ Carnegie Mellon University \\ Pittsburgh, PA 15213 USA \\ <dougb, aberger, lafferty> Ccs.cmu.edu
}

\begin{abstract}
This paper introduces new methods based on exponential families for modeling the correlations between words in text and speech. While previous work assumed the effects of word co-occurrence statistics to be constant over a window of several hundred words, we show that their influence is nonstationary on a much smaller time scale. Empirical data drawn from English and Japanese text, as well as conversational speech, reveals that the "attraction" between words decays exponentially, while stylistic and syntactic contraints create a "repulsion" between words that discourages close co-occurrence. We show that these characteristics are well described by simple mixture models based on twostage exponential distributions which can be trained using the EM algorithm. The resulting distance distributions can then be incorporated as penalizing features in an exponential language model.
\end{abstract}

\section{Introduction}

One of the fundamental characteristics of language, viewed as a stochastic process, is that it is highly nonstationary. Throughout a written document and during the course of spoken conversation, the topic evolves, effecting local statistics on word occurrences. The standard trigram model disregards this nonstationarity, as does any stochastic grammar which assigns probabilities to sentences in a contextindependent fashion.

\footnotetext{
"Research supported in part by NSF grant IRI9314969, DARPA AASERT award DAAH04-95-1-0475, and the ATR Interpreting Telecommunications Research Laboratories.
}

Stationary models are used to describe such a dynamic source for at least two reasons. The first is convenience: stationary models require a relatively small amount of computation to train and to apply. The second is ignorance: we know so little about how to model effectively the nonstationary characteristics of language that we have for the most part completely neglected the problem. From a theoretical standpoint, we appeal to the Shannon-McMillanBreiman theorem (Cover and Thomas, 1991) whenever computing perplexities on test data; yet this result only rigorously applies to stationary and ergodic sources.

To allow a language model to adapt to its recent context, some researchers have used techniques to update trigram statistics in a dynamic fashion by creating a cache of the most recently seen $n$-grams which is smoothed together (typically by linear interpolation) with the static model; see for example (Jelinek et al., 1991; Kuhn and de Mori, 1990). Another approach, using maximum entropy methods similar to those that we present here, introduces a parameter for trigger pairs of mutually informative words, so that the occurrence of certain words in recent context boosts the probability of the words that they trigger (Rosenfeld, 1996). Triggers have also been incorporated through different methods (Kuhn and de Mori, 1990; Ney, Essen, and Kneser, 1994). All of these techniques treat the recent context as a "bag of words," so that a word that appears, say, five positions back makes the same contribution to prediction as words at distances of 50 or 500 positions back in the history.

In this paper we introduce new modeling techniques based on exponential families for capturing the long-range correlations between occurrences of words in text and speech. We show how for both written text and conversational speech, the empirical distribution of the distance between trig- 
ger words exhibits a striking behavior in which the "attraction" between words decays exponentially, while stylistic and syntactic constraints create a "repulsion" between words that discourages close cooccurrence.

We have discovered that this observed behavior is well described by simple mixture models based on two-stage exponential distributions. Though in common use in queueing theory, such distributions have not, to our knowledge, been previously exploited in speech and language processing. It is remarkable that the behavior of a highly complex stochastic process such as the separation between word cooccurrences is well modeled by such a simple parametric family, just as it is surprising that Zipf's law can so simply capture the distribution of word frequencies in most languages.

In the following section we present examples of the empirical evidence for the effects of distance. In Section 3 we outline the class of statistical models that we propose to model this data. After completing this work we learned of a related paper (Niesler and Woodland, 1997) which constructs similar models. In Section 4 we present a parameter estimation algorithm, based on the EM algorithm, for determining the maximum likelihood estimates within the class. In Section 5 we explain how distance models can be incorporated into an exponential language model, and present sample perplexity results we have obtained using this class of models.

\section{The Empirical Evidence}

The work described in this paper began with the goal of building a statistical language model using a static trigram model as a "prior," or default distribution, and adding certain features to a family of conditional exponential models to capture some of the nonstationary features of text. The features we used were simple "trigger pairs" of words that were chosen on the basis of mutual information. Figure 1 provides a small sample of the $41,263(s, t)$ trigger pairs used in most of the experiments we will describe.

In earlier work, for example (Rosenfeld, 1996), the distance between the words of a trigger pair $(s, t)$ plays no role in the model, meaning that the "boost" in probability which $t$ receives following its trigger $s$ is independent of how long ago $s$ occurred, so long as $s$ appeared somewhere in the history $H$, a fixedlength window of words preceding $t$. It is reasonable to expect, however, that the relevance of a word $s$ to the identity of the next word should decay as $s$ falls

\begin{tabular}{||l|l||}
\hline$s$ & $t$ \\
\hline Ms. & her \\
changes & revisions \\
energy & gas \\
committee & representative \\
board & board \\
lieutenant & colonel \\
AIDS & AIDS \\
Soviet & missiles \\
undernater & diving \\
patients & drugs \\
television & airqaves \\
Voyager & Meptune \\
medical & surgical \\
I & me \\
Gulf & Gulf \\
\hline
\end{tabular}

Figure 1: A sample of the 41,263 trigger pairs extracted from the 38 million word Wall Street Journal corpus.

\begin{tabular}{|c|c|}
\hline$s$ & $t$ \\
\hline UN & $\begin{array}{l}\text { 安保理 } \\
\text { Security Council }\end{array}$ \\
\hline $\begin{array}{l}\text { 昌力 } \\
\text { electricity }\end{array}$ & $\begin{array}{l}\text { キロワ\%ト時 } \\
\text { kilogatt }\end{array}$ \\
\hline $\begin{array}{l}\text { 選举 } \\
\text { election }\end{array}$ & $\begin{array}{l}\text { 小簧举区 } \\
\text { small electoral district }\end{array}$ \\
\hline $\begin{array}{l}\text { 生糸 } \\
\text { silk }\end{array}$ & $\begin{array}{l}\text { 㛺蔽 } \\
\text { cocoon }\end{array}$ \\
\hline $\begin{array}{l}\text { 地樓 } \\
\text { court }\end{array}$ & $\begin{array}{l}\text { 憼役 } \\
\text { imprisonment }\end{array}$ \\
\hline $\begin{array}{l}\text { ハンカリリー } \\
\text { Hungary }\end{array}$ & $\begin{array}{l}\text { ブルガリ } \\
\text { Bulgaria }\end{array}$ \\
\hline $\begin{array}{l}\text { 日本航空 } \\
\text { Japan Air }\end{array}$ & $\begin{array}{l}\text { 究輸 } \\
\text { to fly cargo }\end{array}$ \\
\hline $\begin{array}{l}\text { 判決 } \\
\text { sentence }\end{array}$ & $\begin{array}{l}\text { 求刑 } \\
\text { proposed punishment }\end{array}$ \\
\hline 移植 & 生体 \\
\hline transplant & organ \\
\hline $\begin{array}{l}\text { 森林 } \\
\text { forest }\end{array}$ & $\begin{array}{l}\text { 古緍 } \\
\text { vastepaper }\end{array}$ \\
\hline $\begin{array}{l}\text { コンピューター } \\
\text { computer }\end{array}$ & $\begin{array}{l}\text { 水 } \\
\text { host }\end{array}$ \\
\hline
\end{tabular}

Figure 2: A sample of triggers extracted from the 33 million word Nikkei corpus.

further and further back into the context. Indeed, there are tables in (Rosenfeld, 1996) which suggest that this is so, and distance-dependent "memory weights" are proposed in (Ney, Essen, and Kneser, 1994). We decided to investigate the effect of distance in more detail, and were surprised by what we found. 

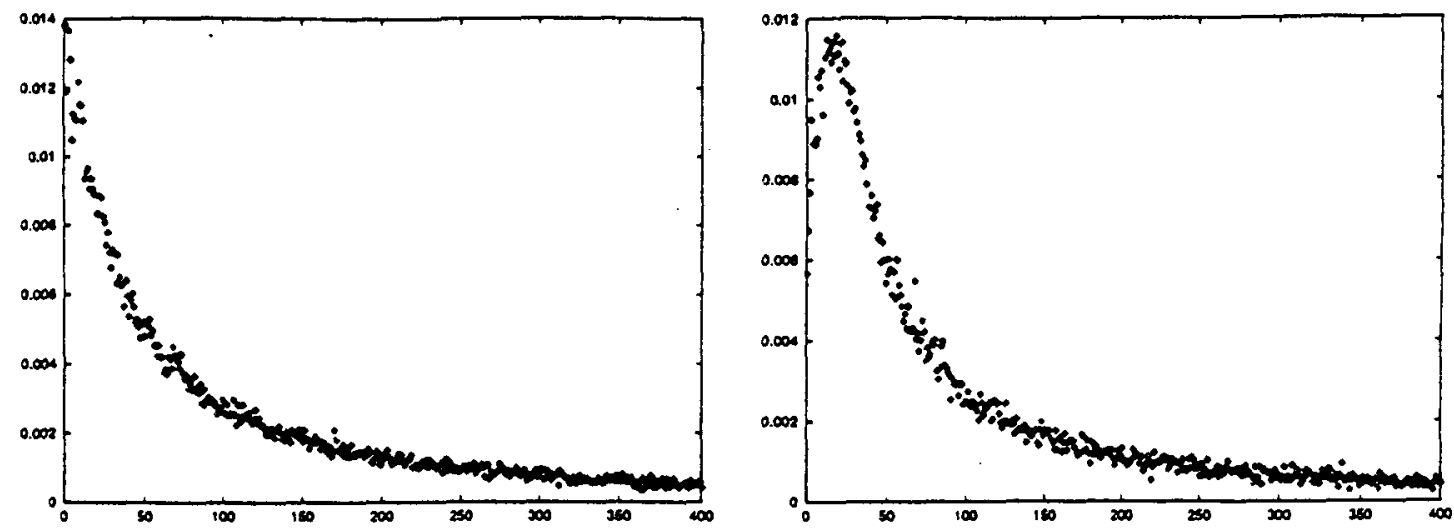

Figure 3: The observed distance distributions-collected from five million words of the Wall Street Journal corpus-for one of the non-self trigger groups (left) and one of the self trigger groups (right). For a given distance $0 \leq k \leq 400$ on the $x$-axis, the value on the $y$-axis is the empirical probability that two trigger words within the group are separated by exactly $k+2$ words, conditional on the event that they co-occur within a 400 word window. (We exclude separation of one or two words because of our use of distance models to improve upon trigrams.)

The set of 41,263 trigger pairs was partitioned into 20 groups of non-self triggers $(s, t), s \neq t$, such as (Soviet, Kremlin's), and 20 groups of self triggers $(s, s)$, such as (business, business). Figure 3 displays the empirical probability that a word $t$ appears for the first time $k$ words after the appearance of its mate $s$ in a trigger pair $(s, t)$, for two representative groups.

The curves are striking in both their similarities and their differences. Both curves seem to have more or less flattened out by $N=400$, which allows us to make the approximating assumption (of great practical importance) that word-triggering effects may be neglected after several hundred words. The most prominent distinction between the two curves is the peak near $k=25$ in the self trigger plots; the nonself trigger plots suggest a monotonic decay. The shape of the self trigger curve, in particular the rise between $k=1$ and $k \approx 25$, reflects the stylistic and syntactic injunctions against repeating a word too soon. This effect, which we term the lexical exclusion principle, does not appear for non-self triggers. In general, the lexical exclusion principle seems to be more in effect for uncommon words, and thus the peak for such words is shifted further to the right.

While the details of the curves vary depending on the particular triggers, this behavior appears to be universal. For triggers that appear too few times in the data for this behavior to exhibit itself, the curves emerge when the counts are pooled with those from a collection of other rare words. An example of this law of large numbers is shown in Figure 4.

These empirical phenomena are not restricted to the Wall Street Journal corpus. In fact, we have observed similar behavior in conversational speech and Japanese text. The corresponding data for self triggers in the Switchboard data (Godfrey, Holliman, and $\mathrm{McDaniel}, 1992)$, for instance, exhibits the same bump in $p(k)$ for small $k$, though the peak is closer to zero. The lexical exclusion principle, then, seems to be less applicable when two people are conversing, perhaps because the stylistic concerns of written communication are not as important in conversation. Several examples from the Switchboard and Nikkei corpora are shown in Figure 5.

\section{Exponential Models of Distance}

The empirical data presented in the previous section exhibits three salient characteristics. First is the decay of the probability of a word $t$ as the distance $k$ from the most recent occurrence of its mate $s$ increases. The most important (continuous-time) distribution with this property is the single-parameter exponential family

$$
p_{\mu}(x)=\mu e^{-\mu x} .
$$

(We'll begin by showing the continuous analogues of the discrete formulas we actually use, since they are simpler in appearance.) This family is uniquely characterized by the memoryless property that the probability of waiting an additional length of time $\Delta t$ is independent of the time elapsed so far, and 

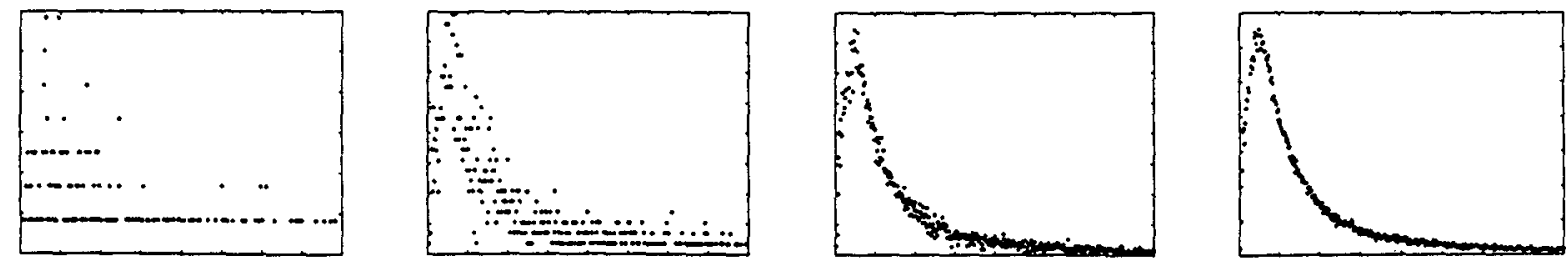

Figure 4: The law of large numbers emerging for distance distributions. Each plot shows the empirical distance curve for a collection of self triggers, each of which appears fewer than 100 times in the entire 38 million word Wall Street Journal corpus. The plots include statistics for $10,50,500$, and all 2779 of the self triggers which occurred no more than 100 times each.
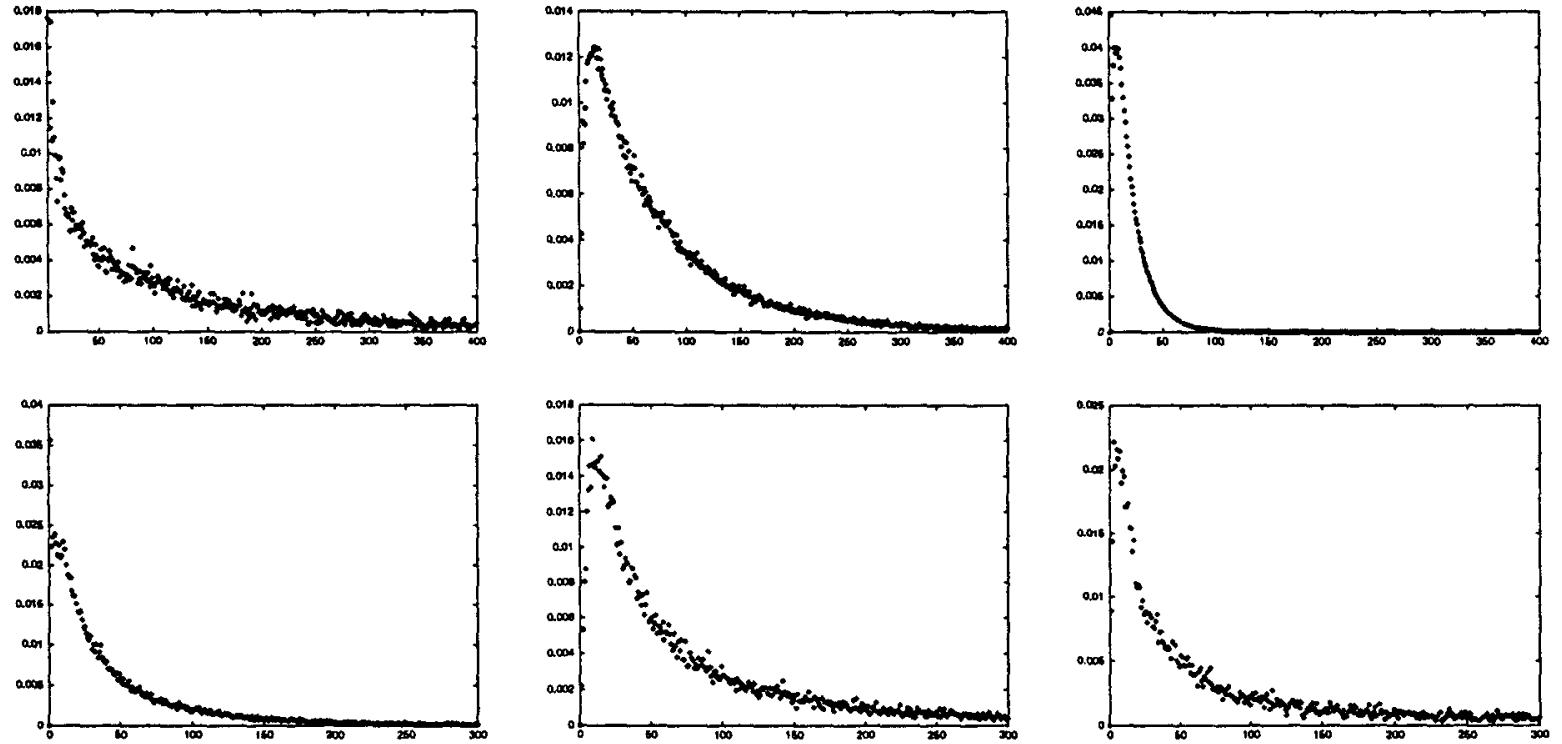

Figure 5: Empirical distance distributions of triggers in the Japanese Nikkei corpus, and the Switchboard corpus of conversational speech. Upper row: All non-self (left) and self triggers (middle) appearing fewer than 100 times in the Nikkei corpus, and the curve for the possessive particle $の$ (right). Bottom row: self trigger UH (left), YOU-KNOW (middle), and all self triggers appearing fewer than 100 times in the entire Switchboard corpus (right).

the distribution $p_{\mu}$ has mean $1 / \mu$ and variance $1 / \mu^{2}$. This distribution is a good candidate for modeling non-self triggers.

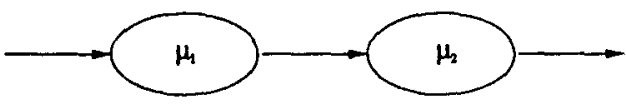

Figure 6: A two-stage queue

The second characteristic is the bump between 0 and 25 words for self triggers. This behavior appears when two exponential distributions are arranged in serial, and such distributions are an important tool in the "method of stages" in queueing theory (Kleinrock, 1975). The time it takes to travel through two service facilities arranged in serial, where the first provides exponential service with rate $\mu_{1}$ and the second provides exponential service with rate $\mu_{2}$, is simply the convolution of the two exponentials:

$$
\begin{aligned}
p_{\mu_{1}, \mu_{2}}(x) & =\mu_{1} \mu_{2} \int_{0}^{x} e^{-\mu_{1} t} e^{-\mu_{2}(x-t)} d t \\
& =\frac{\mu_{1} \mu_{2}}{\mu_{2}-\mu_{1}}\left(e^{-\mu_{2} x}-e^{-\mu_{2} x}\right) \quad \mu_{1} \neq \mu_{2} .
\end{aligned}
$$

The mean and variance of the two-stage exponential $p_{\mu_{1}, \mu_{2}}$ are $1 / \mu_{1}+1 / \mu_{2}$ and $1 / \mu_{1}^{2}+1 / \mu_{2}^{2}$ respectively. As $\mu_{1}$ (or, by symmetry, $\mu_{2}$ ) gets large, the peak shifts towards zero and the distribution approaches the single-parameter exponential $p_{\mu_{2}}$ (by 
symmetry, $\left.p_{\mu_{1}}\right)$. A sequence of two-stage models is shown in Figure 7.

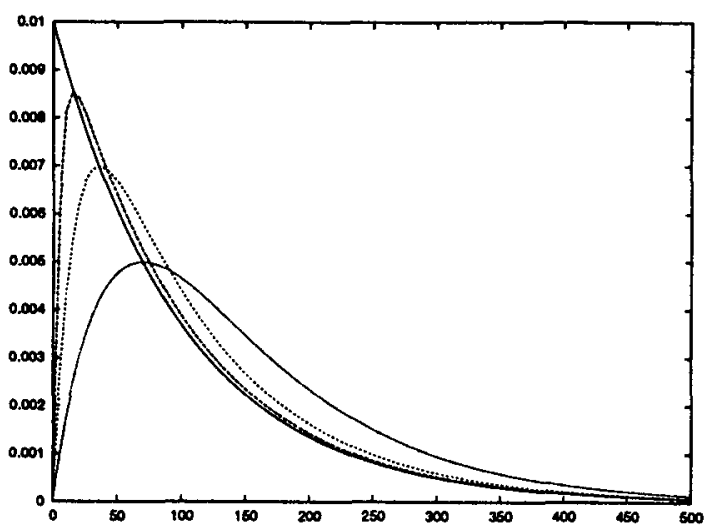

Figure 7: A sequence of two-stage exponential models $p_{\mu_{1}, \mu_{2}}(x)$ with $\mu_{1}=0.01,0.02,0.06,0.2, \infty$ and $\mu_{2}=0.01$.

The two-stage exponential is a good candidate for distance modeling because of its mathematical properties, but it is also well-motivated for linguistic reasons. The first queue in the two-stage model represents the stylistic and syntactic constraints that prevent a word from being repeated too soon. After this waiting period, the distribution falls off exponentially, with the memoryless property. For nonself triggers, the first queue has a waiting time of zero, corresponding to the absence of linguistic constraints against using $t$ soon after $s$ when the words $s$ and $t$ are different. Thus, we are directly modeling the "lexical exclusion" effect and long-distance decay that have been observed empirically.

The third artifact of the empirical data is the tendency of the curves to approach a constant, positive value for large distances. While the exponential distribution quickly approaches zero, the empirical data settles down to a nonzero steady-state value.

Together these three features suggest modeling distance with a three-parameter family of distributions:

$$
p_{\mu_{1}, \mu_{2}, c}(x)=\gamma^{-1}\left(p_{\mu_{1}, \mu_{2}}(x)+c\right)
$$

where $c>0$ and $\gamma$ is a normalizing constant. Rather than a continuous-time exponential, we use the discrete-time analogue

$$
p_{\mu}(k)=\left(1-e^{-\mu}\right) e^{-\mu k} .
$$

In this case, the two-stage model becomes the discrete-time convolution

$$
p_{\mu_{1}, \mu_{2}}(k)=\sum_{t=0}^{k} p_{\mu_{1}}(t) p_{\mu_{2}}(k-t) .
$$

Remark. It should be pointed out that there is another parametric family that is an excellent candidate for distance models, based on the first two features noted above. This is the Gamma distribution

$$
p_{\alpha, \beta}(x)=\frac{\beta^{\alpha}}{\Gamma(\alpha)} x^{\alpha-1} e^{-\beta x}
$$

This distribution has mean $\alpha / \beta$ and variance $\alpha / \beta^{2}$ and thus can afford greater flexibility in fitting the empirical data. For Bayesian analysis, this distribution is appropriate as the conjugate prior for the exponential parameter $\mu$ (Gelman et al., 1995). Using this family, however, sacrifices the linguistic interpretation of the two-stage model.

\section{Estimating the Parameters}

In this section we present a solution to the problem of estimating the parameters of the distance models introduced in the previous section. We use the maximum likelihood criterion to fit the curves. Thus, if $\theta \in \Theta$ represents the parameters of our model, and $\tilde{p}(k)$ is the empirical probability that two triggers appear a distance of $k$ words apart, then we seek to maximize the log-likelihood

$$
\mathcal{L}(\theta)=\sum_{k \geq 0} \tilde{p}(k) \log p_{\theta}(k) .
$$

First suppose that $\left\{p_{\theta}\right\}_{\theta \in \Theta}$ is the family of continuous one-stage exponential models $p_{\mu}(k)=\mu e^{-\mu k}$. In this case the maximum likelihood problem is straightforward: the mean is the sufficient statistic for this exponential family, and its maximum likelihood estimate is determined by

$$
\mu=\frac{1}{\sum_{k \geq 0} k \tilde{p}(k)}=\frac{1}{E_{\tilde{p}}[k]} .
$$

In the case where we instead use the discrete model $p_{\mu}(k)=\left(1-e^{-\mu}\right) e^{-\mu k}$, a little algebra shows that the maximum likelihood estimate is then

$$
\mu=\log \left(1+\frac{1}{E_{\tilde{p}}[k]}\right) .
$$

Now suppose that our parametric family $\left\{p_{\theta}\right\}_{\theta \in \Theta}$ is the collection of two-stage exponential models; the log-likelihood in this case becomes

$$
\mathcal{L}\left(\mu_{1}, \mu_{2}\right)=\sum_{k \geq 0} \tilde{p}(k) \log \left(\sum_{j=0}^{k} p_{\mu_{1}}(j) p_{\mu_{2}}(k-j)\right) .
$$

Here it is not obvious how to proceed to obtain the maximum likelihood estimates. The difficulty is that there is a sum inside the logarithm, and direct differentiation results in coupled equations for $\mu_{1}$ and 
$\mu_{2}$. Our solution to this problem is to view the convolving index $j$ as a hidden variable and apply the EM algorithm (Dempster, Laird, and Rubin, 1977). Recall that the interpretation of $j$ is the time used to pass through the first queue; that is, the number of words used to satisfy the linguistic constraints of lexical exclusion. This value is hidden given only the total time $k$ required to pass through both queues.

Applying the standard EM argument, the difference in log-likelihood for two parameter pairs $\left(\mu_{1}^{\prime}, \mu_{2}^{\prime}\right)$ and $\left(\mu_{1}, \mu_{2}\right)$ can be bounded from below as

$$
\begin{aligned}
\mathcal{L}\left(\mu^{\prime}\right)-\mathcal{L}(\mu)=\sum_{k \geq 0} \tilde{p}(k) \log \left(\frac{p_{\mu_{1}^{\prime}, \mu_{2}^{\prime}}(k)}{p_{\mu_{1}, \mu_{2}}(k)}\right) \\
\quad \geq \sum_{k \geq 0} \tilde{p}(k) \sum_{j=0}^{k} p_{\mu_{1}, \mu_{2}}(j \mid k) \log \left(\frac{p_{\mu_{1}^{\prime}, \mu_{2}^{\prime}}(k, j)}{p_{\mu_{1}, \mu_{2}}(k, j)}\right) \\
\equiv \mathcal{A}\left(\mu^{\prime}, \mu\right)
\end{aligned}
$$

where

$$
p_{\mu_{1}, \mu_{2}}(k, j)=p_{\mu_{1}}(j) p_{\mu_{2}}(k-j)
$$

and

$$
p_{\mu_{1}, \mu_{2}}(j \mid k)=\frac{p_{\mu_{1}, \mu_{2}}(k, j)}{p_{\mu_{1}, \mu_{2}}(k)} .
$$

Thus, the auxiliary function $\mathcal{A}$ can be written as

$$
\begin{aligned}
\mathcal{A}\left(\mu^{\prime}, \mu\right)= & \log \left(1-e^{-\mu_{1}^{\prime}}\right)+\log \left(1-e^{-\mu_{2}^{\prime}}\right) \\
& -\mu_{1}^{\prime} \sum_{k \geq 0} \tilde{p}(k) \sum_{j=0}^{k} j p_{\mu_{1}, \mu_{2}}(j \mid k) \\
& -\mu_{2}^{\prime} \sum_{k \geq 0} \tilde{p}(k) \sum_{j=0}^{k}(k-j) p_{\mu_{1}, \mu_{2}}(j \mid k) \\
& +\operatorname{constant}(\mu) .
\end{aligned}
$$

Differentiating $\mathcal{A}\left(\mu^{\prime}, \mu\right)$ with respect to $\mu_{i}^{\prime}$, we get the EM updates

$$
\begin{gathered}
\mu_{1}^{\prime}=\log \left(1+\frac{1}{\sum_{k \geq 0} \tilde{p}(k) \sum_{j=0}^{k} j p_{\mu_{1}, \mu_{2}}(j \mid k)}\right) \\
\mu_{2}^{\prime}=\log \left(1+\frac{1}{\sum_{k \geq 0} \tilde{p}(k) \sum_{j=0}^{k}(k-j) p_{\mu_{1}, \mu_{2}}(j \mid k)}\right)
\end{gathered}
$$

Remark. It appears that the above updates require $O\left(N^{2}\right)$ operations if a window of $N$ words is maintained in the history. However, using formulas for the geometric series, such as $\sum_{k=0}^{\infty} k x^{k}=x /(1-x)^{2}$, we can write the expectation $\sum_{j=0}^{k} j p_{\mu_{1}, \mu_{2}}(j \mid k)$ in closed form. Thus, the updates can be calculated in linear time.

Finally, suppose that our parametric family $\left\{p_{\theta}\right\}_{\theta \in \Theta}$ is the three-parameter collection of twostage exponential models together with an additive constant:

$$
p_{\mu_{1}, \mu_{2}, c}(k)=\gamma^{-1}\left(p_{\mu_{1}, \mu_{2}}(k)+c\right)
$$

Here again, the maximum likelihood problem can be solved by introducing a hidden variable. In particular, by setting $\alpha=\frac{c}{c+N^{-1}}$ we can express this model as a mixture of a two-stage exponential and a uniform distribution:

$$
p_{\mu_{1}, \mu_{2}, \alpha}(k)=(1-\alpha) p_{\mu_{1}, \mu_{2}}(k)+\alpha\left(\frac{1}{N}\right) \text {. }
$$

Thus, we can again apply the EM algorithm to determine the mixing parameter $\alpha$. This is a standard application of the EM algorithm, and the details are omitted.

In summary, we have shown how the EM algorithm can be applied to determine maximum likelihood estimates of the three-parameter family of distance models $\left\{p_{\mu_{1}, \mu_{2}, \alpha}\right\}$ of distance models. In Figure 8 we display typical examples of this training algorithm at work.

\section{A Nonstationary Language Model}

To incorporate triggers and distance models into a long-distance language model, we begin by constructing a standard, static backoff trigram model (Katz, 1987), which we will denote as $q\left(w_{0} \mid w_{-1}, w_{-2}\right)$. For the purposes of building a model for the Wall Street Journal data, this trigram model is quickly trained on the entire 38-million word corpus. We then build a family of conditional exponential models of the general form

$$
\begin{aligned}
& p(w \mid H)= \\
& \frac{1}{Z(H)} \exp \left(\sum_{i} \lambda_{i} f_{i}(H, w)\right) q\left(w \mid w_{-1}, w_{-2}\right)
\end{aligned}
$$

where $H=w_{-1}, w_{-2}, \ldots, w_{-N}$ is the word history, and $Z(H)$ is the normalization constant

$$
Z(H)=\sum_{w} \exp \left(\sum_{i} \lambda_{i} f_{i}(H, w)\right) q\left(w \mid w_{-1}, w_{-2}\right)
$$

The functions $f_{i}$, which depend both on the word history $H$ and the word being predicted, are called features, and each feature $f_{i}$ is assigned a weight $\lambda_{i}$. In the models that we built, feature $f_{i}$ is an indicator function, testing for the occurrence of a trigger pair $\left(s_{i}, t_{i}\right)$ :

$$
f_{i}(H, w)= \begin{cases}1 & \text { if } s_{i} \in H \text { and } w=t_{i} \\ 0 & \text { otherwise. }\end{cases}
$$

The use of the trigram model as a default distribution (Csiszár, 1996) in this manner is new in language modeling. (One might also use the term prior, although $q(w \mid H)$ is not a prior in the strict Bayesian sense.) Previous work using maximum entropy methods incorporated trigram constraints as 

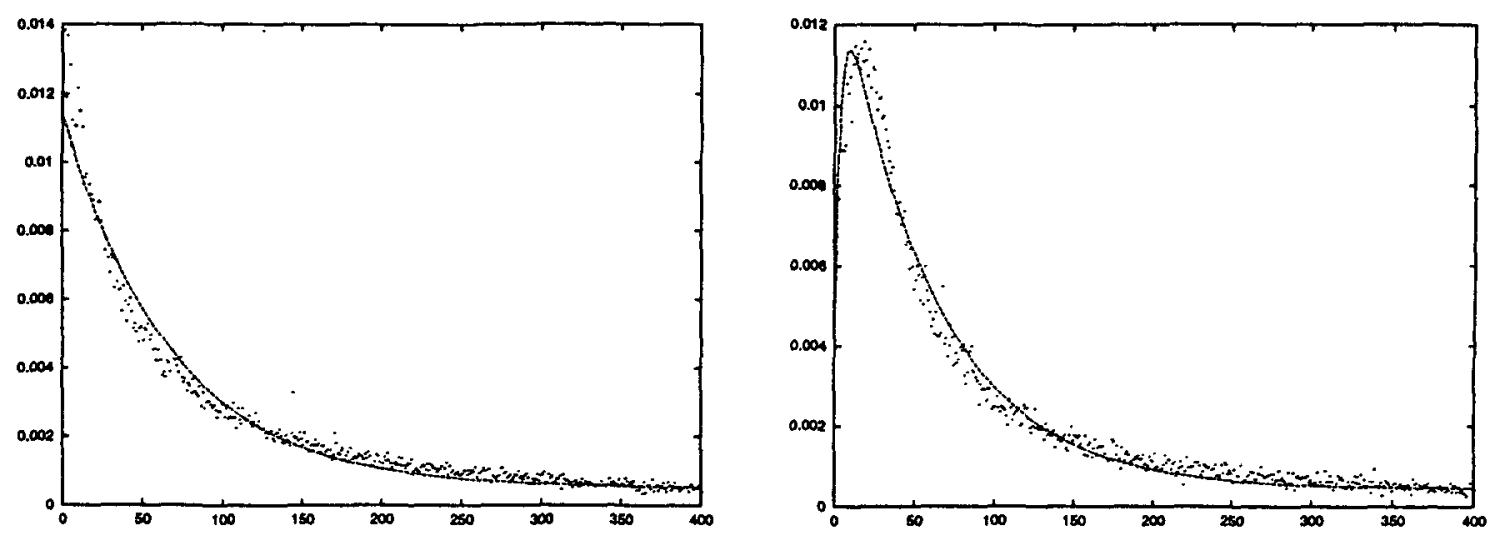

Figure 8: The same empirical distance distributions of Figure 2 fit to the three-parameter mixture model $p_{\mu_{1}, \mu_{2}, \alpha}$ using the EM algorithm. The dashed line is the fitted curve. For the non-self trigger plot $\mu_{1}=7$, $\mu_{2}=0.0148$, and $\alpha=0.253$. For the self trigger plot $\mu_{1}=0.29, \mu_{2}=0.0168$, and $\alpha=0.224$.

explicit features (Rosenfeld, 1996), using the uniform distribution as the default model. There are several advantages to incorporating trigrams in this way. The trigram component can be efficiently constructed over a large volume of data, using standard software or including the various sophisticated techniques for smoothing that have been developed. Furthermore, the normalization $Z(H)$ can be computed more efficiently when trigrams appear in the default distribution. For example, in the case of trigger features, since

$$
Z(H)=1+\sum_{i} \delta\left(s_{i} \in H\right)\left(e^{\lambda_{i}}-1\right) q\left(t_{i} \mid w_{-1}, w_{-2}\right)
$$

the normalization involves only a sum over those words that are actively triggered. Finally, assuming robust estimates for the parameters $\lambda_{i}$, the resulting model is essentially guaranteed to be superior to the trigram model. The training algorithm we use for estimating the parameters is the Improved Iterative Scaling (IIS) algorithm introduced in (Della Pietra, Della Pietra, and Lafferty, 1997).

To include distance models in the word predictions, we treat the distribution on the separation $k$ between $s_{i}$ and $t_{i}$ in a trigger pair $\left(s_{i}, t_{i}\right)$ as a prior. Suppose first that our distance model is a simple one-parameter exponential, $p\left(k \mid s_{i} \in H, w=t_{i}\right)=$ $\mu_{i} e^{-\mu_{i} k}$. Using Bayes' theorem, we can then write

$$
\begin{aligned}
p(w & \left.=t_{i} \mid s_{i} \in H, s_{i}=w_{-k}\right) \\
& =\frac{p\left(w=t_{i} \mid s_{i} \in H\right) p\left(k \mid s_{i} \in H, w=t_{i}\right)}{p\left(k \mid s_{i} \in H\right)} \\
& \propto e^{\lambda_{i}-\mu_{i} k} q\left(t_{i} \mid w_{i-1}, w_{i-2}\right) .
\end{aligned}
$$

Thus, the distance dependence is incorporated as a penalizing feature, the effect of which is to discour- age a large separation between $s_{i}$ and $t_{i}$. A simjlar interpretation holds when the two-stage mixture models $p_{\mu_{1}, \mu_{2}, \alpha}$ are used to model distance, but the formulas are more complicated.

In this fashion, we first trained distance models using the algorithm outlined in Section 4. We then incorporated the distance models as penalizing features, whose parameters remained fixed, and proceeded to train the trigger parameters $\lambda_{i}$ using the IIS algorithm. Sample perplexity results are tabulated in Figure 9.

One important aspect of these results is that because a smoothed trigram model is used as a default distribution, we are able to bucket the trigger features and estimate their parameters on a modest amount of data. The resulting calculation takes only several hours on a standard workstation, in comparison to the machine-months of computation that previous language models of this type required.

The use of distance penalties gives only a small improvement, in terms of perplexity, over the baseline trigger model. However, we have found that the benefits of distance modeling can be sensitive to configuration of the trigger model. For example, in the results reported in Table 9, a trigger is only allowed to be active once in any given context. By instead allowing multiple occurrences of a trigger $s$ to contribute to the prediction of its mate $t$, both the perplexity reduction over the baseline trigram and the relative improvements due to distance modeling are increased. 


\begin{tabular}{|l|c|c|}
\hline Experiment & Perplexity & Reduction \\
\hline Baseline: trigrams trained on 5M words & 170 & - \\
\hline Trigram prior + 41,263 triggers & 145 & $14.7 \%$ \\
\hline Same as above + distance modeling & 142 & $16.5 \%$ \\
\hline \hline Baseline: trigrams trained on 38M words & 107 & - \\
\hline Trigram prior + 41,263 triggers & 92 & $14.0 \%$ \\
\hline Same as above + distance modeling & 90 & $15.9 \%$ \\
\hline
\end{tabular}

Figure 9: Models constructed using trigram priors. Training the larger model required about 10 hours on a DEC Alpha workstation.

\section{Conclusions}

We have presented empirical evidence showing that the distribution of the distance between word pairs that have high mutual information exhibits a striking behavior that is well modeled by a threeparameter family of exponential models. The properties of these co-occurrence statistics appear to be exhibited universally in both text and conversational speech. We presented a training algorithm for this class of distance models based on a novel application of the EM algorithm. Using a standard backoff trigram model as a default distribution, we built a class of exponential language models which use nonstationary features based on trigger words to allow the model to adapt to the recent context, and then incorporated the distance models as penalizing features. The use of distance modeling results in an improvement over the baseline trigger model.

\section{Acknowledgement}

We are grateful to Fujitsu Laboratories, and in particular to Akira Ushioda, for providing access to the Nikkei corpus within Fujitsu Laboratories, and assistance in extracting Japanese trigger pairs.

\section{References}

Berger, A., S. Della Pietra, and V. Della Pietra. 1996. A maximum entropy approach to natural language processing. Computational Linguistics, 22(1):39-71.

Cover, T.M. and J.A. Thomas. 1991. Elements of In. formation Theory. John Wiley.

Csiszár, I. 1996. Maxent, mathematics, and information theory. In K. Hanson and R. Silver, editors, Maximum Entropy and Bayesian Methods. Kluwer Academic Publishers.
Della Pietra, S., V. Della Pietra, and J. Lafferty. 1997. Inducing features of random fields. IEEE Trans. on Pattern Analysis and Machine Intelligence, 19(3), March.

Dempster, A.P., N.M. Laird, and D.B. Rubin. 1977. Maximum likelihood from incomplete data via the EM algorithm. Journal of the Royal Statistical Society, 39(B):1-38.

Gelman, A., J. Carlin, H. Stern, and D. Rubin. 1995. Bayesian Data Analysis. Chapman \& Hall, London.

Godfrey, J., E. Holliman, and J. McDaniel. 1992. SWITCHBOARD: Telephone speech corpus for research and development. In Proc. ICASSP.92.

Jelinek, F., B. Merialdo, S. Roukos, and M. Strauss. 1991. A dynamic language model for speech recognition. In Proceedings of the DARPA Speech and Natural Language Workshop, pages 293-295, February.

Katz, S. 1987. Estimation of probabilities from sparse data for the langauge model component of a speech recognizer. IEEE Transactions on Acoustics, Speech and Signal Processing, ASSP-35(3):400-401, March.

Kleinrock, L. 1975. Queueing Systems. Volume 1: Theory. Wiley, New York.

Kuhn, R. and R. de Mori. 1990. A cache-based natural language model for speech recognition. IEEE Trans. on Pattern Analysis and Machine Intelligence, 12:570-583.

Ney, H., U. Essen, and R. Kneser. 1994. On structuring probabilistic dependencies in stochastic language modeling. Computer Speech and Language, 8:1-38.

Niesler, T. and P. Woodland. 1997. Modelling wordpair relations in a category-based language model. In Proceedings of ICASSP.97, Munich, Germany, April.

Rosenfeld, R. 1996. A maximum entropy approach to adaptive statistical language modeling. Computer Speech and Language, 10:187-228. 